\title{
Thrombotic Responses of Endothelial Outgrowth Cells to Protein-Coated Surfaces
}

\author{
Deirdre E.J. Anderson ${ }^{a}$ Kathryn A. McKenna ${ }^{a}$ Jeremy J. Glynn ${ }^{a} \quad$ Ulla Marzec $^{b}$ \\ Stephen R. Hanson ${ }^{a, b}$ Monica T. Hinds ${ }^{a, b}$ \\ a Department of Biomedical Engineering, Oregon Health and Science University, Portland, Oreg., and \\ b Oregon National Primate Research Center, Oregon Health and Science University, Beaverton, Oreg., USA
}

\section{Key Words}

Activated protein C . Endothelial cells · Endothelial outgrowth cells · Extracellular matrix · Expanded polytetrafluoroethylene - Thrombosis - Tissue engineering · Vascular grafts

\begin{abstract}
There is significant clinical need for viable small-diameter vascular grafts. While there are many graft biomaterials in development, few have been clinically successful. Evaluation of grafts with a clinically relevant model is needed to drive development. This work examined extracellular matrix coatings on the thrombotic phenotype of endothelial outgrowth cells (EOCs). EOCs were tested on flat plates and tubular grafts. Flat plate studies examined collagen I, collagen IV, fibronectin and a-elastin coatings. EOCs attached or proliferated more readily on collagen I and fibronectin surfaces as determined by total DNA. The production of activated protein C (APC) by EOCs was also dependent on the surface coating, with collagen I and fibronectin displaying a higher activity than both collagen IV and a-elastin on flat plate studies. Based on these results, only collagen I and fibronectin coatings were tested on expanded polytetrafluoroethylene (ePTFE) in the ex vivo model. Tubular samples showed significantly greater tissue factor pathway inhibitor gene expression on collagen I than
\end{abstract}

on fibronectin. Platelet adhesion was not significantly different, but EOCs on collagen I produced significantly lower APC than on fibronectin, suggesting that differences exist between the flat plate and tubular cultures. Overall, while the hemostatic phenotype of EOCs displayed some differences, cell responses were largely independent of the matrix coating. EOCs adhered strongly to both fibronectin- and collagen-l-coated ePTFE grafts under ex vivo (100 $\mathrm{ml} / \mathrm{min})$ flow conditions suggesting the usefulness of this clinically relevant cell source, testing modality, and shunt model for future work examining biomaterials and cell conditioning before implantation.

(c) 2015 S. Karger AG, Basel

\section{Abbreviations used in this paper}

APC

EC

ECM

EOC

EPCR

ePTFE

FBS

PBS

TF

TFPI

TM

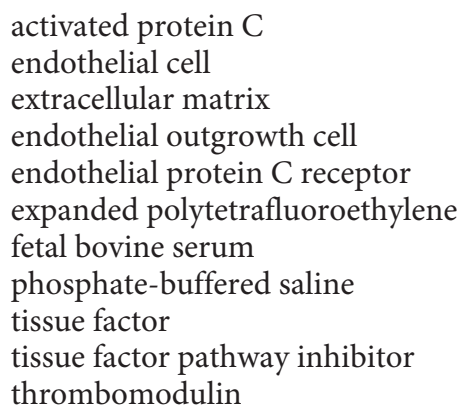

\section{KARGER}

E-Mail karger@karger.com

www.karger.com/cto
C 2015 S. Karger AG, Basel

$1422-6405 / 15 / 1994-0238 \$ 39.50 / 0$ 


\section{Introduction}

Heart disease remains the number one cause of morbidity and mortality in the Western world. As of 2012, an estimated 82.6 million people are affected by cardiovascular disease in the United States [Roger et al., 2012]. Thus, cardiovascular disease has a significant effect on the United States health care system with direct and indirect costs totaling almost 300 billion USD. Despite this profound impact, treatments using vascular grafts have not significantly changed in 30 years, with mammary artery and saphenous vein autografts remaining the gold standard treatment for bypass surgery. Synthetic materials, such as expanded polytetrafluoroethylene (ePTFE) and polyethylene terephthalate (also known as Dacron ${ }^{\circledR}$ ), are easily manufactured with controllable physical properties but lack favorable biological properties. For example, unmodified small-diameter ePTFE grafts exposed to blood flow result in thrombus formation and inflammation but fail to endothelialize after 8 weeks in a canine model [Murray-Wijelath et al., 2004]. These synthetic materials have been successfully used for large grafts $>5 \mathrm{~mm}$ in diameter, but are not appropriate for small-diameter vessels. Two critical determinants of vascular graft success are the prevention of thrombosis and intimal hyperplasia [Zilla et al., 2007]. Many variables modulate the thrombogenic potential of a graft construct, and considerable efforts have been made to develop a synthetic graft with favorable blood-contacting properties [Sarkar et al., 2007]. A strategy to develop a compatible synthetic surface has been to modify the interface properties of the graft by methods including: extracellular matrix (ECM) coating of collagen [Guidoin et al., 1996], incorporating heparin [Olsson et al., 2000; Lin et al., 2004] and antiplatelet factors [van der Lei et al., 1992; Aldenhoff et al., 2001], modifying surface charge and hydrophilicity [Pektok et al., 2009], and endothelialization of the grafts [Deutsch et al., 2009].

Endothelialization of a graft surface is a promising strategy for improving graft hemocompatibility. With this approach, endothelial functions are not simply mimicked, but they are inherently provided by the native regulatory mechanisms. Endothelial cells (ECs) are potent regulators of thrombosis, and the conditions under which ECs are cultured can push the cellular response towards pro- or antithrombotic pathways. EC regulation of thrombosis acts through three distinct yet interrelated systems: (1) the coagulation cascade, which is managed through surface-bound receptors on the EC surface, (2) secretion of factors into the circulation to regulate fibri-

Thrombotic Responses of Endothelial Outgrowth Cells nolysis and vascular tone, and (3) cell-mediated responses involving blood cells, such as leukocytes and platelets, where ECs utilize both membrane-bound and secreted factors to regulate vascular injury repair [McGuigan and Sefton, 2007]. Understanding how ECs mediate thrombosis and coagulation may help to determine their performance on vascular graft biomaterials. Under normal conditions, ECs maintain a delicate balance between pro- and anticoagulant responses. As reviewed elsewhere, ECs acting through both released factors, such as tissue factor pathway inhibitor (TFPI), and membrane-bound factors, including tissue factor (TF), endothelial protein $\mathrm{C}$ receptor (EPCR), and thrombomodulin (TM), play a critical role in the initiation, propagation, and termination of the coagulation cascade [McGuigan and Sefton, 2007]. Specifically, TF is a prothrombotic marker involved in the activation of factor $\mathrm{X}$ to $\mathrm{Xa}$. TFPI is an upstream inhibitor of this activation. TM is an antithrombotic marker which catalyzes the activation of protein $\mathrm{C}$ to activated protein $\mathrm{C}$ (APC). APC will inactivate factor Va and VIIIa, limiting thrombin generation. EPCR is an EC surface maker that also enhances protein $\mathrm{C}$ activation. CD39 is another EC surface marker that is involved in limiting platelet activation. Thus the expression and activity of these proand anticoagulant factors must be characterized to determine cell phenotype.

Potential autologous cell sources for endothelialization may be derived from adipose tissue, bone marrow, veins, and arteries [Salacinski et al., 2001]. While these sources may yield viable ECs, they also require more invasive surgical procedures for isolation. Peripheral blood endothelial outgrowth cells (EOCs) are a promising potential autologous cell source for tissue-engineered vascular grafts [Kaushal et al., 2001; Griese et al., 2003], which require a simple venipuncture and removal of a relatively small volume of blood. Endothelial progenitor cells, isolated from peripheral blood and possessing 'EClike' properties, were first described by Asahara et al. [1997]. EOCs or late outgrowth cells were later characterized in 2000 by Lin et al. [2000]. EOCs expand rapidly in vitro and are phenotypically similar to mature ECs [Hinds et al., 2008; Tura et al., 2013]. This cell population has been shown to stain positive for typical EC markers of von Willebrand factor, CD31, VE-cadherin, vascular endothelial growth factor-receptor 2, TM, and E-selectin, yet have reduced endothelial nitric oxide synthase expression relative to ECs [Hinds et al., 2008].

In order to test the performance of EOCs on a clinically relevant ePTFE graft, the surface must first be coated with a cytophillic agent, such as an ECM protein. In 
vivo, ECM is important for providing mechanical support, adding strength and resilience to the arterial wall, and regulating cellular functions. The ECM, specifically the basement membrane, is the primary support for the vascular endothelium [Davis and Senger, 2005] and maintains a stable, functional monolayer of ECs [Funk et al., 2010]. A stable basement membrane provides antiproliferative cues to ECs [Kalluri, 2003]. In response to injury, cells lay down provisional ECM proteins, including collagen I and fibronectin. In this study, the role of ECM coatings on the hemostatic activity of EOCs on flat and tubular surfaces was investigated.

Previous work has established a model for testing tubular surfaces by using ex vivo chronic femoral arteriovenous shunts to assess the effects of surfaces on thrombus formation. This well-established model permits excellent control of relevant variables, including blood flow rate (arterial vs. venous), blood flow geometry (e.g., steady, unidirectional vs. disturbed flow), the nature of the thrombogenic surface, and the coagulability of the blood through giving or avoiding anticoagulant therapy [Hanson et al., 1985; Torem et al., 1988; Krupski et al., 1993]. As a result, outcomes have proven to be highly reproducible and the model has become a recognized means for evaluating mechanisms of thrombosis and the effects of therapeutic interventions [Hanson et al., 1985; Torem et al., 1988; Cadroy et al., 1989; Krupski et al., 1993].

This study used a clinically relevant vascular graft model of endothelialized ePTFE grafts [Deutsch et al., 2009], in which the luminal surface of 4-mm-diameter ePTFE grafts were coated with an ECM protein and endothelialized. We hypothesized that collagen IV, an important ECM protein of the basement membrane, would induce an anticoagulant phenotype in EOCs [Dudash et al., 2012] compared to ECM proteins, collagen I, a-elastin, and fibronectin, which have been used as biomaterials for vascular grafts [Berglund et al., 2004; Hinds et al., 2006; Seidlits et al., 2011]. These proteins have been used in a variety of cell-based tissue-engineering applications but without a comprehensive understanding of the effects the ECM may have on the thrombotic properties of the cells. As reviewed by McGuigan and Sefton [2007], surface coatings are frequently examined for cell attachment or proliferation, but considerably less work has examined the phenotype and thrombogenicity of the cells with various surface coatings or their coagulation potential using whole, non-anticoagulated blood. To give a thorough evaluation of the effects that the ECM coating can have on the EOC phenotype, cells were characterized using in vitro analysis of TF, TFPI, EPCR, CD39, and TM gene expression, functional activity responses of APC, and ex vivo platelet and fibrinogen accumulation. These studies improved the understanding of how ECM components affect EOC thrombotic responses in flat plate and tubular configurations. Understanding the cellular response to the ECM proteins will aid in determining the best formulation to create a beneficial environment for cell-seeded, tissue-engineered vascular grafts.

\section{Methods}

\section{EOC Isolation}

Baboon EOCs were isolated from peripheral blood as previously described [Hinds et al., 2008]. Briefly, mononuclear cells were isolated with density centrifugation and seeded on bovine fibronectin (Sigma-Aldrich, St. Louis, Mo., USA) in EC growth media (EGM-2; Lonza, Walkersville, Md., USA) supplemented with fetal bovine serum (FBS; Hyclone, Logan, Utah, USA) for a total of $20 \%$. The cultures were maintained until outgrowth colonies developed, which occurred between 7 and 21 days of culture. EOCs were then passaged and expanded in tissue culture flasks coated with bovine collagen I (MP Biomedicals, Santa Ana, Calif., USA) in EGM-2 supplemented with FBS for a total of 10\%. CD31-positive EOCs were selected at passage 2 using Dynal magnetic beads (Invitrogen, Carlsbad, Calif., USA). The growth medium was EGM-2 supplemented with $10 \%$ FBS in all studies unless noted otherwise. All cells were used between passage numbers 4 and 6 for each culture.

\section{EOC Flat Plate Culture}

EOCs were plated on non-tissue-culture-treated polystyrene 96-well plates and $35-\mathrm{mm}$ dishes coated with $50-\mathrm{mg} / \mathrm{ml}$ solutions of either collagen I (MP Biomedicals), collagen IV (Rockland Immunochemicals for Research, Gilbertsville, Pa., USA), fibronectin (Sigma-Aldrich), or $\alpha$-elastin (synthesis methods have been described previously [McKenna et al., 2012]). Cells were plated at $3 \times 10^{4}$ cells $/ \mathrm{cm}^{2}$ in growth media for $24 \mathrm{~h}$ followed by $24 \mathrm{~h}$ of culture in high-viscosity media. High-viscosity media consisted of a $15 \%$ solution of $100 \mathrm{~g} / \mathrm{l}$ dextrose and $320 \mathrm{~g} / \mathrm{l}$ dextran from Leuconostoc mesenteroides (molecular weight $=48,000-90,000$; Sigma) in endothelial basal media (Lonza) with 5\% FBS, $1 \%$ penicillinstreptomycin-fungizone, $1 \% \mathrm{~L}$-glutamine, and $0.1 \%$ epidermal growth factor. The viscosity of this medium was $3.5 \mathrm{~Pa} \cdot \mathrm{s}$, designed to match the viscosity of blood in vivo.

\section{Graft Construction}

Grafts were constructed such that they could be used for in vitro and ex vivo shunt studies. Small-diameter (4-mm), thin-walled, non-ringed ePTFE vascular grafts (W.L. Gore, Flagstaff, Ariz., USA) were cut to $7 \mathrm{~cm}$ in length and coated with a thin layer of silicone glue on the last $0.5 \mathrm{~cm}$ of each end. Graft ends were cuffed with silicone tubing (Technical Products, Inc., Oxford, Ga., USA) by gluing the tubing to the outer ePTFE surface. The aim of the silicone tubing connected to the ePTFE graft was to create a seamless luminal transition between the graft and tubing materials. The joined ends were reinforced by adding additional silicone glue to the outer surface of the junctions along with an outer stabilizing layer of 2-gauge heat shrink tubing (Small Parts, Inc., Logansport, Ind., USA; fig. 1a). 


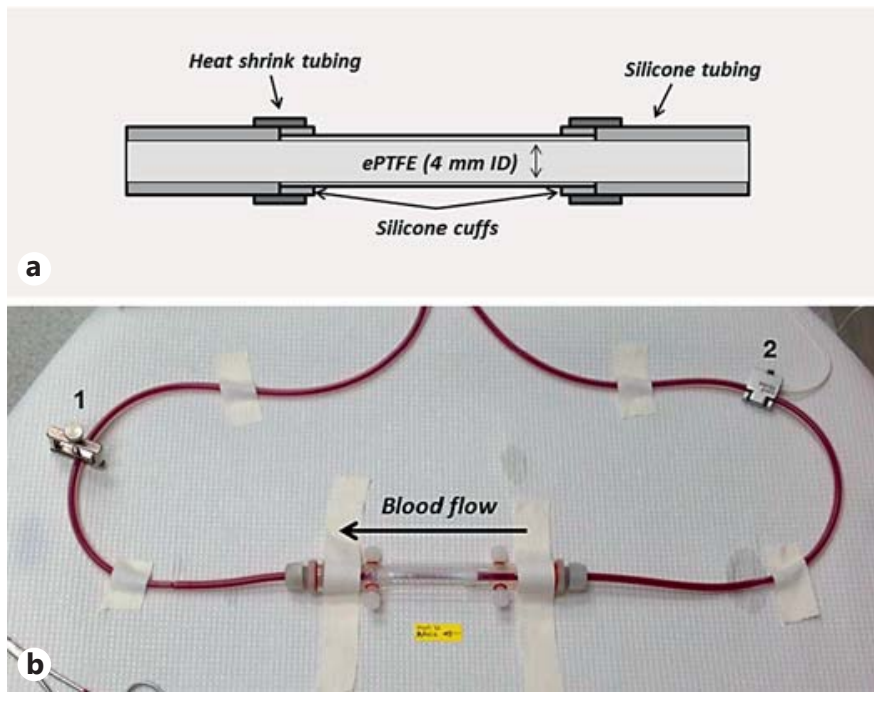

Fig. 1. Ex vivo shunt study setup. Cross-sectional diagram of the graft construction allowing for smooth luminal transitions between the ePTFE and silicone shunt tubing (a). Image of the ex vivo flow loop with the tubular ePTFE construct in a biochamber (b). The clamp, labeled 1, is used to maintain a flow rate at $100 \mathrm{ml} /$ min measured by the flow probe, labeled 2 .

\section{Graft Coating}

To facilitate protein adsorption, the ePTFE graft surface was first permeabilized by infusing $95 \%$ ethanol through the pores of the graft to wet the graft surface. Ethanol was then replaced with water and further rinsed with phosphate-buffered saline (PBS) for fibronectin coatings or $0.02 \mathrm{~N}$ acetic acid in water for collagen-Imodified grafts. Protein solutions of $4 \mathrm{mg} / \mathrm{ml}$ bovine collagen I (MP Biomedicals) or $1 \mathrm{mg} / \mathrm{ml}$ bovine fibronectin (Sigma-Aldrich) were immediately pushed through the pores of the ePTFE until the outer surface was completely saturated with solution. The distal end of the silicone tubing was then rinsed with PBS for fibronectin coating or $0.02 \mathrm{~N}$ acetic acid in water for collagen I. Concentrations of these proteins were chosen based on the maximum concentration possible given the solubility of the proteins. The collagen was stained with the Oregon Green ${ }^{\circledR} 488$ protein labeling kit (Invitrogen) and observed with a fluorescent microscope to ensure an even coating (data not shown).

\section{EOC Seeding on Protein-Modified ePTFE Grafts}

EOCs were seeded onto ePTFE grafts modified with either collagen I or fibronectin in a three-step process. EOCs were suspended in growth media at a concentration of $3 \times 10^{5}$ cells $/ \mathrm{ml}$ and injected into the graft from the distal tubing. The solution was advanced to the proximal end of the graft and perfused through ePTFE to allow for complete cell coverage. Three separate inoculations of $3 \times 10^{5}$ cells $/ \mathrm{ml}$ at 40 -min intervals were added to the graft lumens. The seeded grafts were rotated $120^{\circ}$ between inoculations to ensure an even and confluent cell distribution around the circumference of the graft. The proximal tubing was not exposed to the cell seeding solution or media throughout the conditioning phases.

Thrombotic Responses of Endothelial Outgrowth Cells

\section{In vitro Analyses}

Flat plate samples were analyzed for gene expression and APC production. Tubular constructs were coated and seeded in parallel for ex vivo shunt platelet analysis and in vitro analyses. These in vitro control samples were analyzed for gene expression and APC production as well as assessed with fluorescent microscopy.

\section{Gene Expression}

RNA was isolated using the Mini RNA I isolation and DNA clean-up kits (Zymo Research, Irvine, Calif., USA) for flat plate studies and the RNeasy mini isolation kit (Qiagen, Valencia, Calif., USA) for shunt in vitro controls. The latter RNA was treated with DNase I (Fermentas, Waltham, Mass., USA) and all RNA was converted to cDNA using SuperScript ${ }^{\circledR}$ III reverse transcriptase (Invitrogen). Gene expression was measured using quantitative realtime PCR for TM, EPCR, CD39, TF, and TFPI. In-house-designed primers (table 1) were used with Platinum ${ }^{\circledR} \mathrm{SYBR}^{\circledR}$ Green and ROX reference dye (Invitrogen) to attain amplification curves. Efficiency and specificity of primers used for these studies was confirmed. $2^{-\Delta \Delta C t}$ values for each gene were calculated using the housekeeping gene (GAPDH) and cells cultured on collagen I for the control. Collagen I was chosen for normalization as it is the most common matrix on which ECs and EOCs are cultured. Flat plate studies were normalized to the average $\Delta \mathrm{Ct}$ for collagen I, while ePTFE data were normalized to the collagen I sample run at the same time as the fibronectin sample. The data were transformed to fold change to make the data continuous by subtracting one from the $2^{-\Delta \Delta \mathrm{Ct}}$ for values greater than one and adding one to the negative inverse of the $2^{-\Delta \Delta \mathrm{Ct}}$ for values less than one.

\section{APC Functional Assay}

EOCs were rinsed and incubated for $1 \mathrm{~h}$ at $37^{\circ} \mathrm{C}$ with $5 \mathrm{nM}$ thrombin and $100 \mathrm{nM}$ protein $\mathrm{C}$ (Haematologic Technologies, Essex Junction, Vt., USA) in PBS with calcium and magnesium cations. After $1 \mathrm{~h}$, Refludan (Berlex, Montville, N.J., USA) was added after incubation to inhibit thrombin. Samples were visualized with $1 \mathrm{~mm}$ S-2366 (Chromogenix, Milano, Italy) and compared to an APC standard (Haematologic Technologies). Absorbance was measured at 20 -second intervals over $20 \mathrm{~min}$ at $37^{\circ} \mathrm{C}$ and the concentration of APC determined from the slope of the absorbance curve. Data were normalized to the graft surface area in tubular samples to correct for any difference in sample length.

\section{Total DNA}

The amount of DNA from the EOCs seeded on flat plates was measured with the Quanti-iT ${ }^{\mathrm{TM}}$ PicoGreen ${ }^{\circledR}$ assay (Invitrogen) according to the manufacturer's protocol.

\section{Fluorescent Staining}

Sections of EOC-seeded grafts were fixed in warm 3.7\% paraformaldehyde and then transferred to wells in a 96-well plate for immunofluorescent staining. Cells were permeablized with $0.1 \%$ Triton X-100 for 10 min to allow for cytoskeletal staining. The graft sections were blocked using Image-iT ${ }^{\circledR}$ FX Signal Enhancer (Invitrogen) for $30 \mathrm{~min}$ followed by $10 \%$ goat serum in PBS for $30 \mathrm{~min}$. Alexa Fluor ${ }^{\circledR} 568$ phalloidin (Invitrogen) diluted 1:100 in PBS with $1 \%$ bovine serum albumin was applied for 25 min to stain F-actin, followed by DAPI (Invitrogen) diluted 1:10,000 in PBS with 1\% bovine serum albumin for $5 \mathrm{~min}$ to stain nuclei. The semicircular sections were carefully flattened and mounted onto glass slides 


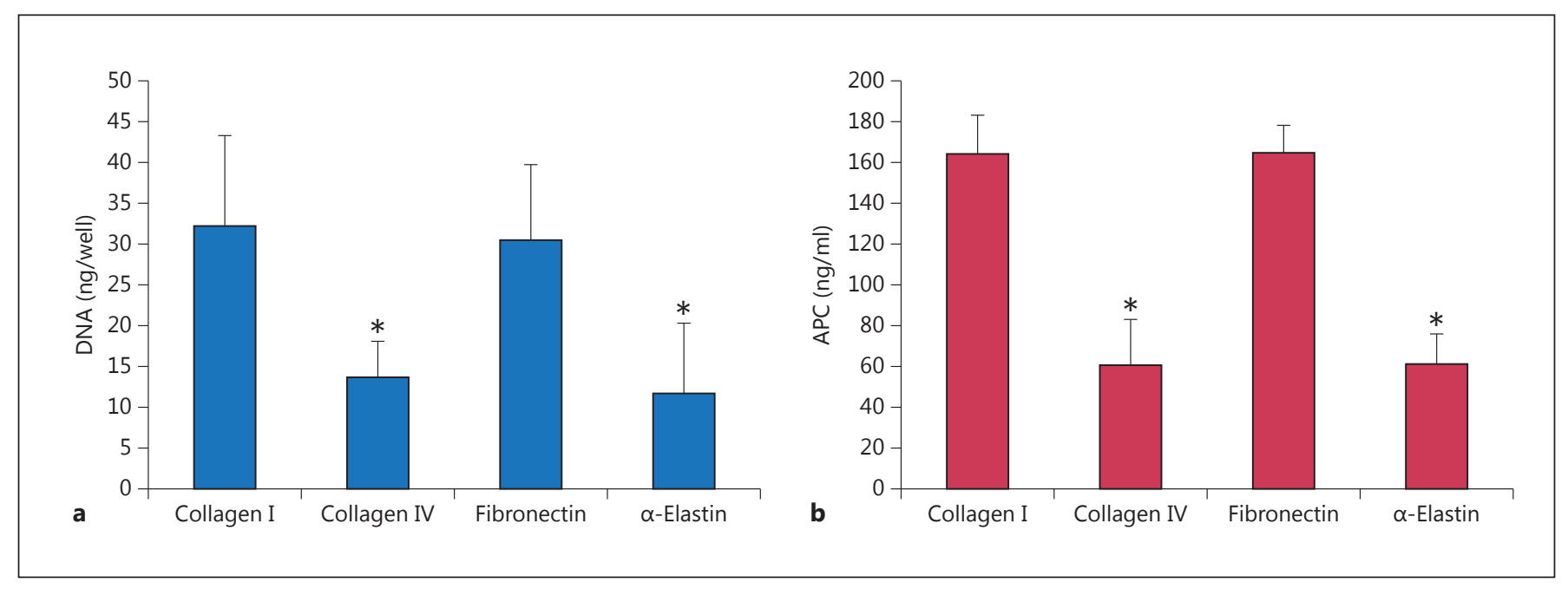

Fig. 2. Flat plate study results. In flat plate studies, EOCs on collagen I and fibronectin coatings had significantly greater total DNA (a) and APC generation (b) compared to collagen IV and $\alpha$-elastin coatings. ${ }^{*}$ p $<0.05$, ANOVA with Tukey's post hoc test.

Table 1. Primer sequences used for gene expression studies

\begin{tabular}{lll}
\hline Gene & Forward primer & Reverse primer \\
\hline GAPDH & CCTCAACGACCACTTTGTCA & TTACTCCTTGGAGGCCATGT \\
TF & CACCGACGAGATTGTGAAGGAT & TTCCCTGCCGGGTAGGAG \\
TFPI & GACTCCGCAATCAACCAAGGT & TGCTGGAGTGAGACACCATGA \\
TM & GGTGGACGGCGAGTGTGTGG & GGTGTTGGGGTCGCAGTCGG \\
EPCR & CACCCTGCAGCAGCTCAATGC & ACATCGCCGTCCACCTGTGC \\
CD39 & AGTGATTCCAAGGTCCCAGCACC & TCCTGAGCAACCGCATGCCT \\
\hline
\end{tabular}

with ProLong ${ }^{\circledR}$ Gold Antifade (Invitrogen). Samples were allowed to cure at room temperature overnight prior to imaging.

\section{Baboon Arteriovenous ex vivo Shunt Model}

All animals were housed at the Department of Animal Resources of the Oregon National Primate Research Center. All of the experiments were approved by the Institutional Animal Care and Use Committee of the Center and adhered to the principles enumerated in the Guide to the Care and Use of Laboratory Animals prepared by the Committee on the Care and Use of Laboratory Animals of the Institute of Laboratory Animal Resources, National Research Council (International Standard Book, Number 0-30905377-3, 1996). A shunt was surgically implanted between the femoral artery and the vein of a 9- to $12-\mathrm{kg}$ male baboon (Papio anubis). The shunt was maintained with short silicone tubing loops until the time of experimentation. The animal was trained to the testing system such that minimal to no sedation was required during the experiment (ketamine, $5-10 \mathrm{mg} / \mathrm{kg}$ ). The baboon was not treated with anticoagulant or antiplatelet therapies during the ex vivo shunt flow studies. The permanent arteriovenous shunt was elongated with silicone tubing and the endothelialized ePTFE construct (fig. 1b) was centered over a gamma scintillation camera (model 400T Maxi-Camera; GE, Fairfield, Conn., USA), which temporally and spatially measured ${ }^{111}$ In accumulation in 5-min intervals on the graft surface. The graft was isolated in a custombuilt flow biochamber. A flowmeter (Transonic Systems Inc., Ithaca, N.Y., USA) was used to monitor flow rates, which were maintained at $100 \mathrm{ml} / \mathrm{min}$ throughout the study by adjusting a tubing clamp distal to the chamber.

EOC retention under flow conditions was assessed in the ex vivo shunt system. The EOCs at $8 \times 10^{6}$ cells $/ \mathrm{ml}$ were labeled with ${ }^{111} \mathrm{In}(50 \mu \mathrm{Ci})$ in a carbonate buffer with $0.2 \mathrm{M}$ tropolone for $10 \mathrm{~min}$ at $37^{\circ} \mathrm{C}$. Radiolabeled EOCs were seeded onto collagen-I- and fibronectin-modified grafts and 96-well plates for a standard curve. The seeded grafts were incubated in growth media for $24 \mathrm{~h}$ followed by high-viscosity media for $24 \mathrm{~h}$. For each shunt study, the amount of ${ }^{111}$ In of the adherent EOCs was acquired prior to and during blood flow. The amount of ${ }^{111}$ In on a $2-\mathrm{cm}$ central region of the ePTFE graft was converted to cell numbers using the standard curve.

The effect of the ECM coating on the ability of EOCs to regulate thrombus formation was assessed in the ex vivo shunt system. Au- 
Fig. 3. Representative images from EOCs on flat plates coated with adsorbed ECM proteins. Proteins tested were collagen I (a), collagen IV (b), fibronectin (c) and a-elastin (d). Images were taken after $24 \mathrm{~h}$ of culture.
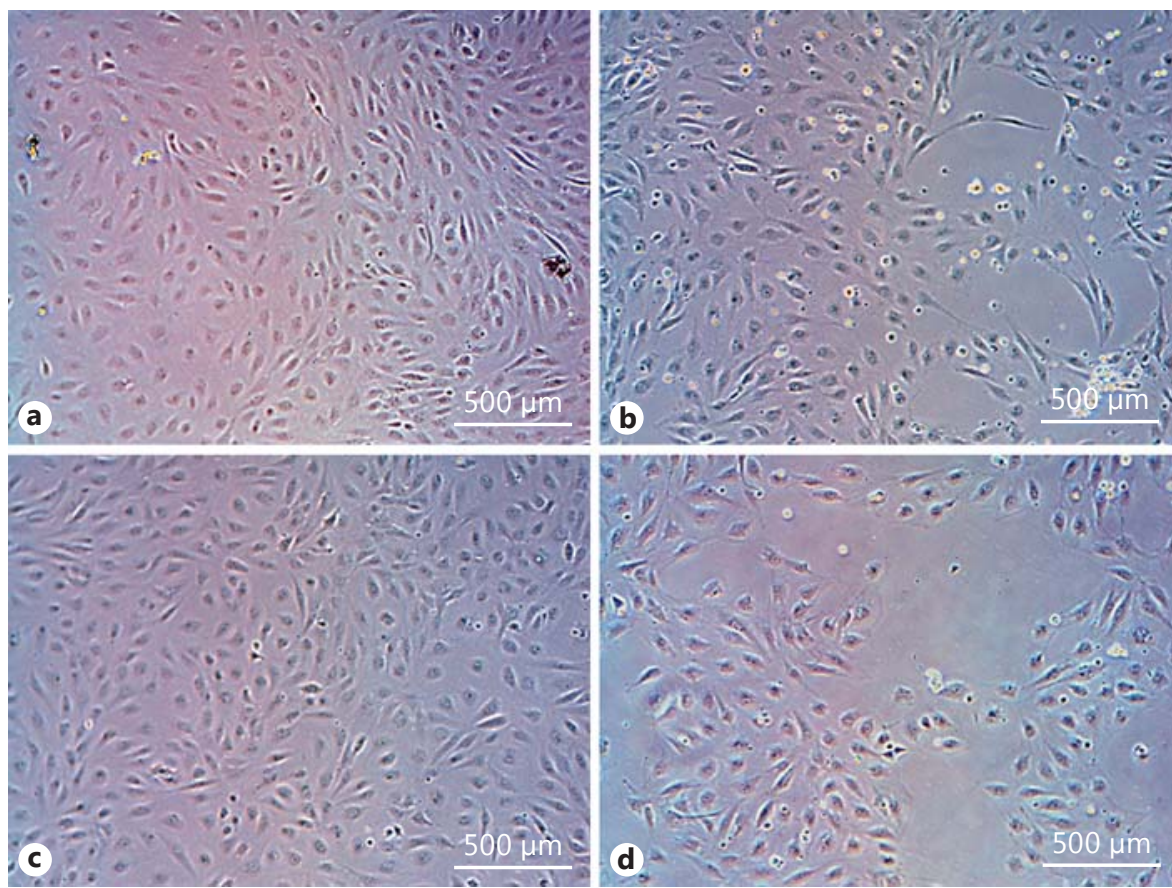

tologous platelets were labeled weekly throughout the duration of the shunt to ensure a strong signal with ${ }^{111}$ In and reinfused into the animals. Homologous fibrinogen was also labeled with ${ }^{125} \mathrm{I}$ and infused into the animal $5 \mathrm{~min}$ prior to flow initiation. As circulating blood flowed through the graft segment, radiolabeled platelets and fibrin accumulated on the graft surface. Thrombus formation was quantified by dynamically measuring platelet deposition and final fibrin deposition. The platelet counts were calculated based on the ${ }^{111}$ In activity using a standard of whole blood prior to each study. The ${ }^{125}$ I-labeled fibrin was measured in a 1480 Wizard Gamma Counter (PerkinElmer, Waltham, Mass., USA) 30 days after the study, once the ${ }^{111}$ In activity had decayed. Platelet and fibrin data were analyzed for a $2-\mathrm{cm}$ central region of the ePTFE graft to avoid any edge effects.

\section{Statistical Analyses}

All data are expressed as means \pm SD. Flat plate gene $(n=4)$ and APC $(n=3)$ data were analyzed with one-way analysis of variance with Tukey's post hoc test. Tubular data $(n=3$ for both gene and APC) were analyzed with paired t tests. In all cases, $\mathrm{p}<0.05$ was used as the measure for statistical significance.

\section{Results}

\section{Flat Plate Studies}

EOCs cultured on adsorbed collagen I, collagen IV, fibronectin, and a-elastin were tested for proliferation, APC production, and gene expression of TM, EPCR, CD39, TF, and TFPI. There was significantly greater DNA content and thus greater cell numbers on the collagen-Iand fibronectin-coated surfaces than on the $\alpha$-elastin- and collagen-IV-coated surfaces (fig. 2a), which was confirmed by visual inspection (fig. 3). Images in figure 3 were taken after $24 \mathrm{~h}$ in culture, but the same cell coverage was seen at $48 \mathrm{~h}$. Gene expression data are reported as fold change compared to collagen I (fig. 4). EOCs on a-elastin showed significantly lower expression of EPCR compared to collagen I. CD39 expression was significantly lower from EOCs on a-elastin than on collagen IV. There were no significant differences in EOC gene expression on the four protein-coated surfaces for TM, TF, and TFPI markers. The APC generated was dependent on surface coating (fig. 2b) with EOCs on collagen I $(164 \pm 20 \mathrm{ng} / \mathrm{ml})$ and fibronectin $(164 \pm 14 \mathrm{ng} / \mathrm{ml})$ displaying significantly higher APC activity than EOCs on both collagen IV (61 \pm 22 $\mathrm{ng} / \mathrm{ml})$ and $\alpha$-elastin $(61 \pm 15 \mathrm{ng} / \mathrm{ml})$. While cell quantities were lower in some samples, this trend remained when APC was normalized by DNA content.

\section{Ex vivo Tubular Studies}

EOCs were successfully seeded onto the lumen of collagen-I- and fibronectin-impregnated ePTFE grafts. Cells formed confluent monolayers as observed through immunofluorescent staining of ePTFE graft sections. Cells on the luminal surface were spread and confluent $48 \mathrm{~h}$ after growth and high-viscosity media conditioning 


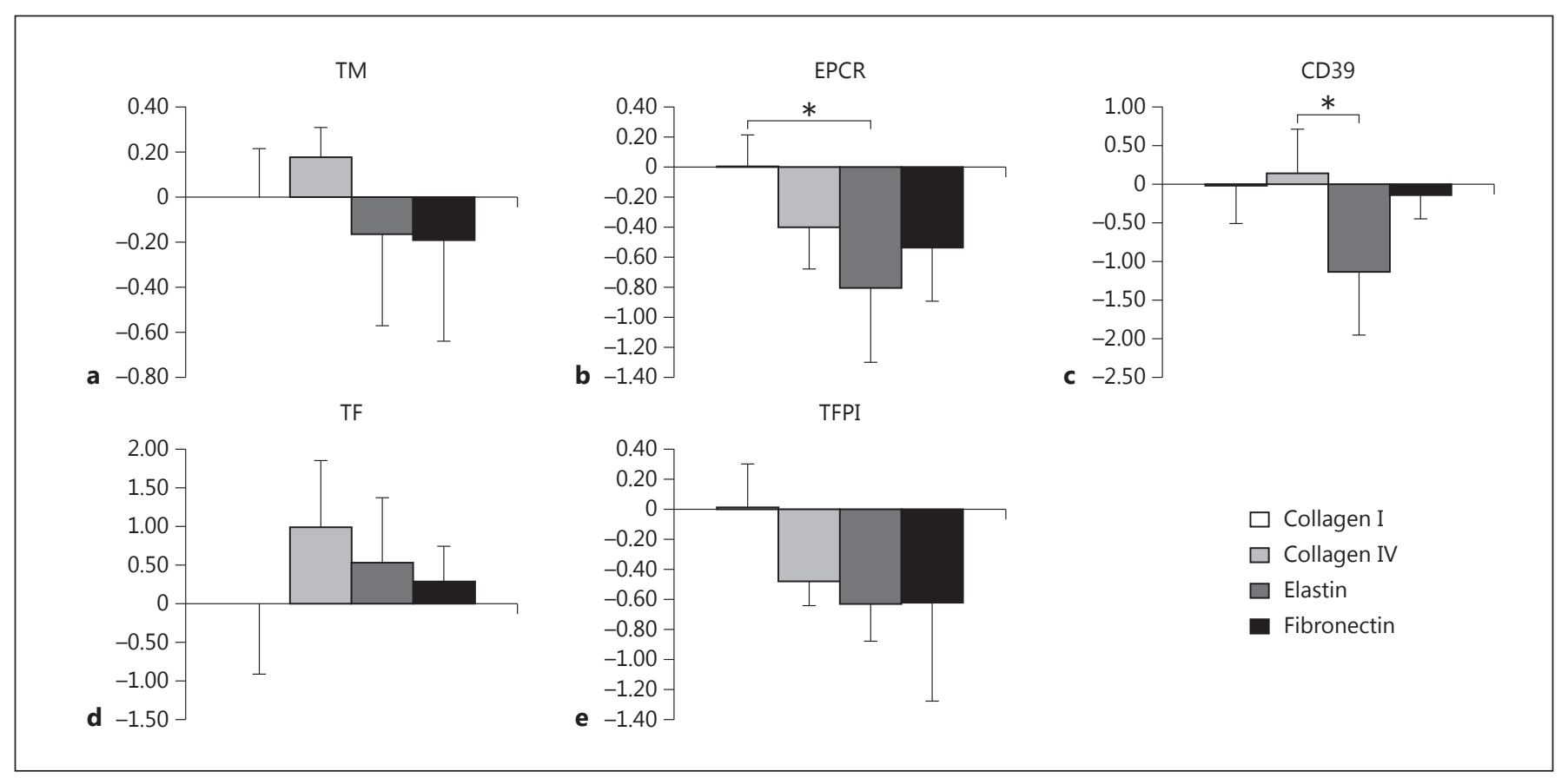

Fig. 4. Gene expression of EOCs on flat plate ECM coatings. Data are shown as a fold increase/decrease compared to EOCs on collagen I. ${ }^{*} \mathrm{p}<0.05$, ANOVA with Tukey's post hoc test. $\mathrm{n}=4$ on each coating.

Table 2. Gene expression of EOCs on ECM-coated ePTFE

\begin{tabular}{|c|c|c|c|c|}
\hline TM & EPCR & CD39 & $\mathrm{TF}$ & TFPI \\
\hline$-0.66 \pm 1.07$ & $-0.27 \pm 0.21$ & $-0.00 \pm 0.02$ & $-1.77 \pm 3.33$ & $-0.33 \pm 0.08^{*}$ \\
\hline
\end{tabular}

(fig. 5a, b). No cells were observed when imaging the external face of the graft sections.

Cell retention was quantified under ex vivo thrombus flow conditions $(100 \mathrm{ml} / \mathrm{min}$ ) for EOCs on both collagenI- and fibronectin-coated ePTFE grafts. The amount of adherent cells initially dropped from the no-flow starting condition but remained stable for $1 \mathrm{~h}$ throughout the duration of a standard shunt study period (fig. 5c). There was no significant difference in cell number between the collagen-I- and fibronectin-coated grafts prior to flow or between 5 and $60 \mathrm{~min}$ of flow.

Gene expression was quantified from EOCs on collagen-I- and fibronectin-modified ePTFE grafts and reported as fold change compared to EOCs on collagen I (table 2). There were no significant differences in gene expression for most of the coagulation gene panel between EOCs on collagen I and fibronectin, with the exception of TFPI. EOCs on collagen I showed significantly higher expression of TFPI compared to EOCs on fibronectin with a $-0.33 \pm 0.08$-fold change.

EOCs on fibronectin produced significantly more APC than EOCs on collagen-I-modified ePTFE grafts (fig. 6a). EOCs on collagen I samples generated $1.13 \pm 0.46 \mathrm{ng} / \mathrm{mm}^{2}$ of APC compared to $1.37 \pm 0.50 \mathrm{ng} / \mathrm{mm}^{2}$ of APC for EOCs on fibronectin-modified ePTFE grafts, which shows a statistically significant increase (paired $t$ test, $\mathrm{p}<0.05$ ).

All study grafts remained patent throughout the ex vivo studies. There was a variable platelet response in the ex vivo model with a range of $0.58 \times 10^{9}$ to $3.00 \times 10^{9}$ platelets adhering to a $2-\mathrm{cm}$ central region at $60 \mathrm{~min}$. 

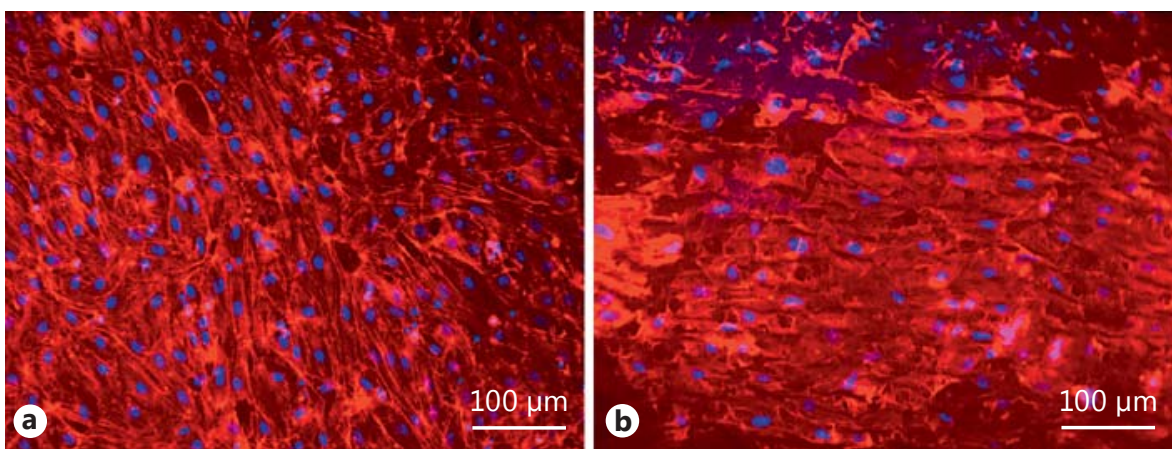

Fig. 5. Representative images of EOCs on ePTFE grafts. EOCs on ePTFE graft surfaces coated with collagen I (a) and fibronectin (b) after $48 \mathrm{~h}$. Nuclei stained with DAPI are shown in blue, and the cytoskeleton (Factin) is stained with Alexa 568 phalloidin shown in red. EOCs attached and spread on the modified ePTFE surface. c The durability test of ${ }^{111}$ In-labeled EOCs on protein-coated ePTFE in the baboon ex vivo shunt system. There was an initial drop (from static to the first 5-min frame) in cell numbers with the initiation of flow, but cells remained stable on the graft through $60 \mathrm{~min}$ at standard flow rates used in the ex vivo shunt studies.
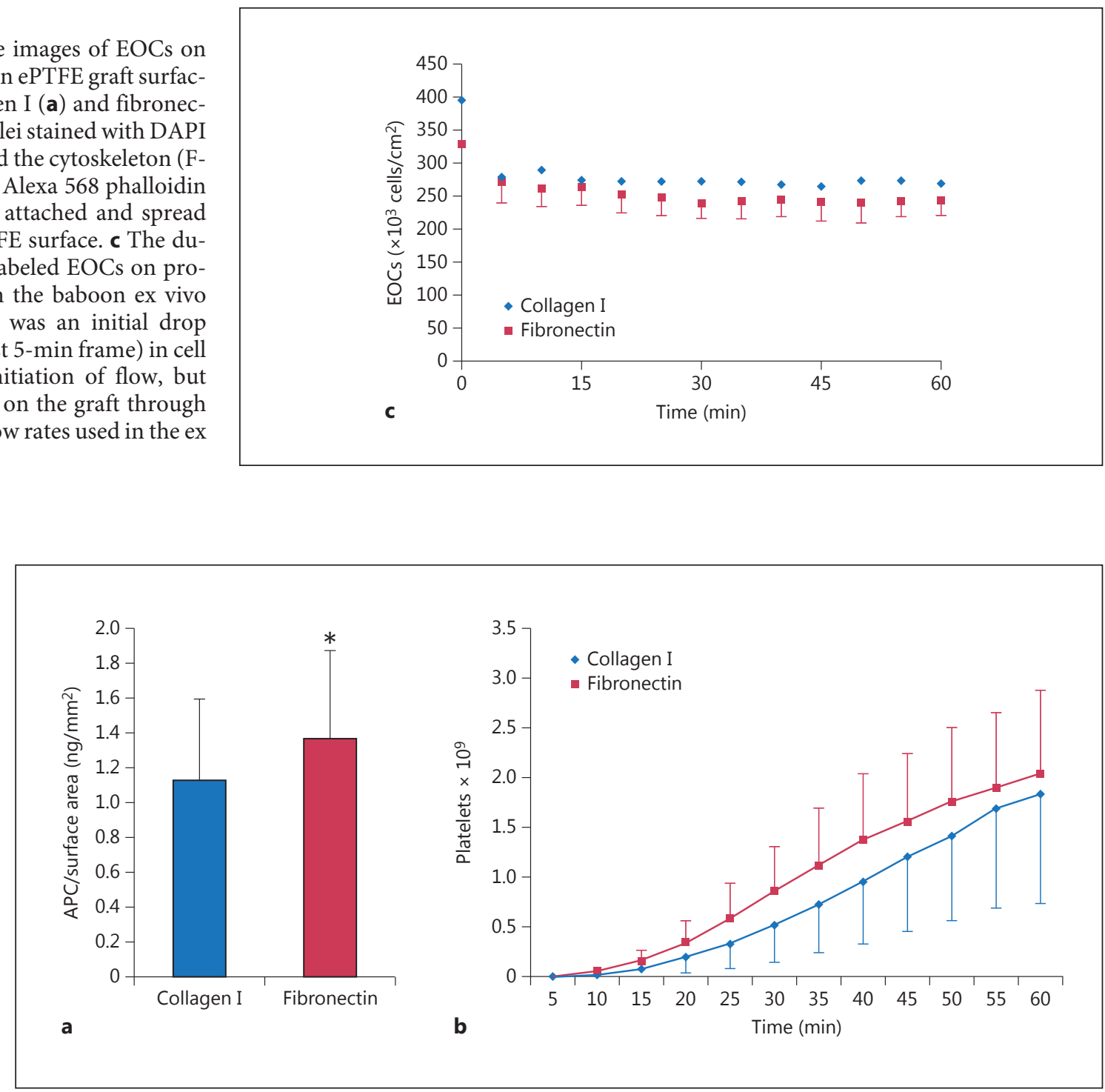

Fig. 6. a APC activity by EOCs on the in vitro ePTFE samples for collagen-I- and fibronectin-modified grafts. ${ }^{*} \mathrm{p}<0.05$, paired t test, $\mathrm{n}=3$. $\mathbf{b}$ Cumulative platelet deposition on the EOC-seeded ePTFE graft surfaces over the $60 \mathrm{~min}$ of the flow period. There were no significant differences between the surface coatings. 
There were no significant differences (paired t test, $\mathrm{n}=3$ ) in platelet numbers between collagen-I- and fibronectinmodified ePTFE grafts with average platelet counts of $1.84 \pm 1.10 \times 10^{9}$ for collagen I and $2.04 \pm 0.84 \times 10^{9}$ for fibronectin at $60 \mathrm{~min}$ (fig. $6 \mathrm{~b}$ ).

The amount of fibrin deposited on the graft surface during the ex vivo shunt studies was measured for a $2-\mathrm{cm}$ region in the central portion of the graft. There were no significant differences (paired t test, $\mathrm{n}=3$ ) between the collagen-I- and fibronectin-modified ePTFE grafts with $1.44 \pm 0.39$ and $1.62 \pm 0.36 \mathrm{mg}$ of fibrin, respectively.

\section{Discussion}

Studying a clinically relevant model of endothelialized vascular grafts is essential for improving the current grafting options when autologous vessels are not available. This work studied not only a clinically relevant material, ePTFE, but also a clinically relevant cell source, EOCs. Since harvesting autologous mature ECs for graft endothelialization is a challenging and time-consuming process that would lead to donor site morbidity, EOCs are an attractive autologous cell source for vascular tissue engineering. They are readily isolated from whole blood, expanded rapidly in vitro, and shown to proliferate on ECM-coated surfaces. Yet, the role of these ECM coatings in regulating the hemostatic functions of EOCs remains largely unknown. ECM composition modulates ECs not only through basic ECM functions like cell attachment and proliferation $[\mathrm{Ku}-$ mar and Krishnan, 2002] but also through more complex signaling that may alter the thrombotic properties of the cells [Prasad and Krishnan, 2005; Prasad et al., 2007]. Determining these changes in EOC thrombogenicity is essential to understand their performance in an in vivo system. Initial studies have characterized the ability of EOCs to regulate the inflammatory and coagulation pathways and their sensitivity to shear stress [Hinds et al., 2008]. Finding an appropriate surface coating to encourage an antithrombotic cell phenotype is critical to further cell-based therapies using artificial graft materials.

Flat plate studies were used to screen ECM proteins of interest: fibronectin, collagen I, collagen IV, and $\alpha$-elastin. The most profound differences seen between EOCs on these surfaces were the cell numbers resulting from DNA quantification at $48 \mathrm{~h}$. There were significantly more cells on the collagen-I- and fibronectin-coated surfaces than on $\alpha$-elastin and collagen IV. The dramatically reduced number of EOCs seen on the collagen IV and $\alpha$-elastin surfaces was unexpected. Since low cell coverage was seen at both 24 and $48 \mathrm{~h}$, and seeded cells were sufficient to provide $100 \%$ confluence, the surfaces either did not support strong cell adhesion or robust proliferation. ECM surface coatings have also altered EOC quantities in previous work. During isolation of EOCs from the mononuclear blood cell fraction, expansion on fibronectin coatings resulted in over twice the number of derived EOCs than cells isolated on collagen IV, collagen I, and laminin [Wang et al., 2004]. This demonstrates that the EOCs are immediately affected by the ECM on which they are plated and that fibronectin may encourage EOC proliferation. Poor integrin-mediated adhesion may have contributed to the low cell numbers observed in the flat plate studies. Previous work found that the activation of specific integrin types on mature ECs was dependent on the specific underlying matrix on which the cells were plated [Orr et al., 2006]. Similarly, the EOCs studied in this work may have been preconditioned by their expansion on collagen-Icoated plates to express a particular set of integrins that were more conducive to adhesion on collagen I and fibronectin compared to collagen IV or a-elastin. Interestingly, vascular wall ECs derived from adult saphenous vein and mammary arteries and grown in subconfluent cultures expressed CD133, an EC precursor marker, and the adhesion of these ECs to ECM-coated ePTFE was dependent on the type of ECM coating. These progenitor-like cells had differential adhesion when seeded on fibronectin, collagen I, collagen IV, and a mix of these proteins, though cell proliferation or resistance to shear stress was not investigated for these individual proteins [Ranjan et al., 2009]. Ranjan et al. [2009] also observed that a combination of the ECM proteins yielded the highest cell adhesion, suggesting the potential benefit of a protein mixture.

The majority of the coagulation gene panel tested in the flat plate study showed no significant differences in expression with the exception of EPCR and CD39. EPCR expression by EOCs was significantly greater on collagen I than on $\alpha$-elastin, and CD39 expression was significantly greater on collagen IV than on $\alpha$-elastin. Trends in TF and TFPI gene expression also suggest an anticoagulant phenotype of EOCs on collagen I. The APC activity assay showed significantly higher protein $\mathrm{C}$ activation by the EOCs on collagen-I- and fibronectin-coated surfaces than on collagen IV or a-elastin. Based on these results, collagen I and fibronectin were chosen as the most promising thromboprotective surface coatings for the tubular ePTFE graft studies. Additionally, given the large quantities of matrix needed for ePTFE coating, collagen I and fibronectin are more practical for future studies due to their lower costs. 
Testing the thromboprotective performance of EOCs on ECM coatings is readily performed on flat plates; however, the poor mimicry of the in vivo environment limits the conclusions that can be drawn regarding graft performance. This suggests the importance of testing in a tubular configuration and the benefit of ex vivo testing, which uses non-anticoagulated blood to more closely model the in vivo clotting response. EOCs seeded on collagen-I- and fibronectin-impregnated ePTFE attached, expanded to confluence, and spread on the lumen of the grafts. Durability studies were conducted on both collagen-I- and fibronectin-modified ePTFE tubular grafts to challenge EOC adhesion and resistance to shear detachment under ex vivo shunt conditions. While cell numbers initially dropped from the no-flow starting condition, they remained stable for $1 \mathrm{~h}$ - the typical ex vivo experimental duration. The initial slight drop in cell numbers was most likely due to loosely adherent cells washing off of the surface of the graft upon the initiation of flow. These results support that the ECM-coated, cell-seeded grafts used here are a robust model for testing endothelialized surfaces in both ex vivo and in vivo environments.

The EOCs on ECM-modified flat plate and ePTFE graft surfaces were evaluated for thrombogenic responses of EOCs in in vitro and ex vivo shunt models, but showed limited differences in gene expression and functional responses to the ECM surface. Since these cells were seeded in the presence of serum, serum proteins may have masked the EOC response to a given surface. Specifically, vitronectin has been shown to adsorb readily to matrix-coated surfaces and mask potential changes that may occur from the ECM coating [McGuigan and Sefton, 2007]. Minor differences were observed between the collagen-I- and fibronectin-coated surfaces. Specifically, the EOCs on fibronectin-modified grafts showed higher APC production values, while EOC expression of the gene for TFPI was significantly lower on the fibronectin samples. While these minor changes in coagulation phenotype did not correlate to a significant ex vivo response, there was a trend toward higher platelet accumulation in the fibronectin samples. This suggests that in this experimental model, the TF pathway may play a larger role in platelet accumulation compared to APC generation, but considerably more work would be needed to test that hypothesis. Alternatively, APC may be more relevant in an in vivo system. The ex vivo system, while dramatically improved from many testing modalities, still represents a limited picture of the coagulation and fibrinolysis potential of the samples. Future work should also more closely examine thrombin generation by EOCs, perhaps through quantifi- cation of TF or factor Xa generation. Additionally, for in vivo implantation, understanding the immunogenic functions of EOCs may be a more critical component of improving this system. Furthermore, these results support the need for more complex testing modalities when looking at tissue-engineering applications. The lack of a correlation between significant changes in TFPI expression and APC production with platelet accumulation reinforces the need for ex vivo and in vivo testing to validate mechanistic results. Additionally, differences in results were observed between cells seeded on flat plates compared to cells on tubular structures. The APC activity assay showed differences in activity between cells on collagen I and fibronectin only on the tubular ePTFE surface studies, but not in the flat plate culture. This indicates that the underlying surface structure is influencing the response of the EOCs, which is an important consideration when translating in vitro results to a tissue-engineering application.

Overall, EOCs on flat plates showed a significantly higher EOC density and APC generation on collagen I and fibronectin compared to collagen IV and $\alpha$-elastin. This was surprising and did not support our original hypothesis that collagen IV would provide the most antithrombotic phenotype. However, the EOCs adhered strongly to tubular constructs coated with fibronectin and collagen I, showing only minor differences in the thrombosis pathway. This work demonstrated that EOCs are a viable and readily isolated autologous cell source that can be used to endothelialize tissue-engineered vascular biomaterials. While this work represents only a first step in improving current vascular grafting technologies, it has paved the way for future work by developing an improved model of endothelialized grafts for ex vivo and in vivo testing. The use of clinically relevant materials and cells will allow for additional testing of other biomaterials, coatings, and cell treatments.

\section{Acknowledgments}

We would like to thank Sawan Hurst and Jennifer Greisel for their excellent technical assistance with the ex vivo shunt animal studies. Also, donations from the Oregon Medical Laser Center for the $\alpha$-elastin and W.L. Gore for the ePTFE are greatly appreciated. This work was funded by the National Institutes of Health grants R01-HL095474 and R01HL103728. It was also supported by the NIH grant OD011092 for the operation of the Oregon National Primate Research Center. This project was also supported by the National Center for Research Resources and the Office of Research Infrastructure Programs of the National Institutes of Health through grant No. RR000163. 


\section{References}

Aldenhoff, Y.B., F.H. van Der Veen, J. ter Woorst, J. Habets, L.A. Poole-Warren, L.H. Koole (2001) Performance of a polyurethane vascular prosthesis carrying a dipyridamole (Persantin) coating on its lumenal surface. J Biomed Mater Res 54: 224-233.

-Asahara, T., T. Murohara, A. Sullivan, M. Silver, R. van der Zee, T. Li, et al. (1997) Isolation of putative progenitor endothelial cells for angiogenesis. Science 275: 964-967.

Berglund, J.D., R.M. Nerem, A. Sambanis (2004) Incorporation of intact elastin scaffolds in tissue-engineered collagen-based vascular grafts. Tissue Eng 10: 1526-1535.

Cadroy, Y., T.A. Horbett, S.R. Hanson (1989) Discrimination between platelet-mediated and coagulation-mediated mechanisms in a model of complex thrombus formation in vivo. J Lab Clin Med 113: 436-448.

Davis, G.E., D.R. Senger (2005) Endothelial extracellular matrix: biosynthesis, remodeling, and functions during vascular morphogenesis and neovessel stabilization. Circ Res 97: 10931107.

Deutsch, M., J. Meinhart, P. Zilla, N. Howanietz, M. Gorlitzer, A. Froeschl, et al. (2009) Longterm experience in autologous in vitro endothelialization of infrainguinal ePTFE grafts. J Vasc Surg 49: 352-362; discussion 362.

Dudash, L.A., F. Kligman, S.M. Sarett, K. KottkeMarchant, R.E. Marchant (2012) Endothelial cell attachment and shear response on biomimetic polymer-coated vascular grafts. Biomed Mater Res A 100: 2204-2210.

Funk, S.D., A. Yurdagul, Jr., J.M. Green, K.A. Jhaveri, M.A. Schwartz, A.W. Orr (2010) Matrix-specific protein kinase A signaling regulates p21-activated kinase activation by flow in endothelial cells. Circ Res 106: 1394-1403.

-Griese, D.P., A. Ehsan, L.G. Melo, D. Kong, L. Zhang, M.J. Mann, et al. (2003) Isolation and transplantation of autologous circulating endothelial cells into denuded vessels and prosthetic grafts: implications for cell-based vascular therapy. Circulation 108: 2710-2715.

- Guidoin, R., Y. Marois, X. Deng, N. Chakfe, M. Marois, R. Roy, et al. (1996) Can collagen impregnated polyester arterial prostheses be recommended as small diameter blood conduits? Asaio J 42: 974-983.

-Hanson, S.R., H.F. Kotze, B. Savage, L.A. Harker (1985) Platelet interactions with Dacron vascular grafts. A model of acute thrombosis in baboons. Arteriosclerosis 5: 595-603.

-Hinds, M.T., M. Ma, N. Tran, A.E. Ensley, S.M. Kladakis, K.B. Vartanian, et al. (2008) Potential of baboon endothelial progenitor cells for tissue engineered vascular grafts. J Biomed Mater Res A 86: 804-812.
Hinds, M.T., R.C. Rowe, Z. Ren, J. Teach, P.C. Wu, S.J. Kirkpatrick, et al. (2006) Development of a reinforced porcine elastin composite vascular scaffold. J Biomed Mater Res A 77: 458-469.

Kalluri, R. (2003) Basement membranes: structure, assembly and role in tumour angiogenesis. Nat Rev Cancer 3: 422-433.

Kaushal, S., G.E. Amiel, K.J. Guleserian, O.M. Shapira, T. Perry, F.W. Sutherland, et al. (2001) Functional small-diameter neovessels created using endothelial progenitor cells expanded ex vivo. Nat Med 7: 1035-1040.

Krupski, W.C., A. Bass, A.B. Kelly, Z.M. Ruggeri, L.A. Harker, S.R. Hanson (1993) Interruption of vascular thrombosis by bolus anti-platelet glycoprotein IIb/IIIa monoclonal antibodies in baboons. J Vasc Surg 17: 294-303; discussion 303-304.

Kumar, T.R., L.K. Krishnan (2002) Fibrin-mediated endothelial cell adhesion to vascular biomaterials resists shear stress due to flow. J Mater Sci Mater Med 13: 751-755.

Lin, P.H., C. Chen, R.L. Bush, Q. Yao, A.B. Lumsden, S.R. Hanson (2004) Small-caliber heparin-coated ePTFE grafts reduce platelet deposition and neointimal hyperplasia in a baboon model. J Vasc Surg 39: 1322-1328.

Lin, Y., D.J. Weisdorf, A. Solovey, R.P. Hebbel (2000) Origins of circulating endothelial cells and endothelial outgrowth from blood. J Clin Invest 105: 71-77.

McGuigan, A.P., M.V. Sefton (2007) The influence of biomaterials on endothelial cell thrombogenicity. Biomaterials 28: 25472571.

McKenna, K.A., K.W. Gregory, R.C. Sarao, C.L. Maslen, R.W. Glanville, M.T. Hinds (2012) Structural and cellular characterization of electrospun recombinant human tropoelastin biomaterials. J Biomater Appl 27: 219-230.

Murray-Wijelath, J., D.J. Lyman, E.S. Wijelath (2004) Vascular graft healing. III. FTIR analysis of ePTFE graft samples from implanted bigrafts. J Biomed Mater Res B Appl Biomater 70: 223-232.

Olsson, P., J. Sanchez, T.E. Mollnes, J. Riesenfeld (2000) On the blood compatibility of endpoint immobilized heparin. J Biomater Sci Polym Ed 11: 1261-1273.

Orr, A.W., M.H. Ginsberg, S.J. Shattil, H. Deckmyn, M.A. Schwartz (2006) Matrix-specific suppression of integrin activation in shear stress signaling. Mol Biol Cell 17: 4686-4697.

Pektok, E., M. Cikirikcioglu, J.C. Tille, A. Kalangos, B.H. Walpoth (2009) Alcohol pretreatment of small-diameter expanded polytetrafluoroethylene grafts: quantitative analysis of graft healing characteristics in the rat abdominal aorta interposition model. Artif Organs 33: 532-537.
Prasad, C.K., L.K. Krishnan (2005) Effect of passage number and matrix characteristics on differentiation of endothelial cells cultured for tissue engineering. Biomaterials 26: 56585667.

Prasad, C.K., C.V. Muraleedharan, L.K. Krishnan (2007) Bio-mimetic composite matrix that promotes endothelial cell growth for modification of biomaterial surface. J Biomed Mater Res A 80: 644-654.

Ranjan, A.K., U. Kumar, A.A. Hardikar, P. Poddar, P.D. Nair (2009) Human blood vesselderived endothelial progenitors for endothelialization of small diameter vascular prosthesis. PLoS One 4: e7718.

Roger, V.L., A.S. Go, D.M. Lloyd-Jones, E.J. Benjamin, J.D. Berry, W.B. Borden, et al. (2012) Heart disease and stroke statistics - 2012 update: a report from the American Heart Association. Circulation 125: e2-e220.

Salacinski, H.J., A. Tiwari, G. Hamilton, A.M. Seifalian (2001) Cellular engineering of vascular bypass grafts: role of chemical coatings for enhancing endothelial cell attachment. Med Biol Eng Comput 39: 609-618.

Sarkar, S., K.M. Sales, G. Hamilton, A.M. Seifalian (2007) Addressing thrombogenicity in vascular graft construction. J Biomed Mater Res B Appl Biomater 82: 100-108.

Seidlits, S.K., C.T. Drinnan, R.R. Petersen, J.B. Shear, L.J. Suggs, C.E. Schmidt (2011) Fibronectin-hyaluronic acid composite hydrogels for three-dimensional endothelial cell culture. Acta Biomater 7: 2401-2409.

Torem, S., P.A. Schneider, S.R. Hanson (1988) Monoclonal antibody-induced inhibition of platelet function: effects on hemostasis and vascular graft thrombosis in baboons. J Vasc Surg 7: 172-180.

Tura, O., E.M. Skinner, G.R. Barclay, K. Samuel, R.C. Gallagher, M. Brittan, et al. (2013) Late outgrowth endothelial cells resemble mature endothelial cells and are not derived from bone marrow. Stem Cells 31: 338-348.

van der Lei, B., H.L. Bartels, P.H. Robinson, W.W. Bakker (1992) Reduced thrombogenicity of vascular prostheses by coating with ADP-ase. Int Angiol 11: 268-271.

Wang, C., C. Jiao, H.D. Hanlon, W. Zheng, R.J. Tomanek, G.C. Schatteman (2004) Mechanical, cellular, and molecular factors interact to modulate circulating endothelial cell progenitors. Am J Physiol Heart Circ Physiol 286: H1985-H1993.

Zilla, P., D. Bezuidenhout, P. Human (2007) Prosthetic vascular grafts: wrong models, wrong questions and no healing. Biomaterials 28: 5009-5027. 